\title{
Carrier heating in disordered conjugated polymers in electric field
}

\author{
Nenad Vukmirovića) and Lin-Wang Wang \\ Computational Research Division, Lawrence Berkeley \\ National Laboratory, Berkeley, CA 94720, USA.
}

\begin{abstract}
The electric field dependence of charge carrier transport and the effect of carrier heating in disordered conjugated polymers were investigated. A parameter-free multiscale methodology consisting of classical molecular dynamics simulation for the generation of the atomic structure, large system electronic structure and electron-phonon coupling constants calculations and the procedure for extracting the bulk polymer mobility, was used. The results suggested that the mobility of a fully disordered poly(3-hexylthiophene) (P3HT) polymer increases with electric field which is consistent with the experimental results on samples of regiorandom P3HT and different from the results on more ordered regioregular P3HT polymers, where the opposite trend is often observed at low electric fields. We calculated the electric field dependence of the effective carrier temperature and showed however that the effective temperature cannot be used to replace the joint effect of temperature and electric field, in contrast to previous theoretical results from phenomenological models. Such a difference was traced to originate from the use of simplified Miller-Abrahams hopping rates in phenomenological models in contrast to our considerations that explicitly take into account the electronic state wave functions and the interaction with all phonon modes.
\end{abstract}

a) Electronic mail: nvukmirovic@lbl.gov 


\section{INTRODUCTION}

Semiconducting conjugated polymers have become the materials of great interest for the use in electronic and optical devices, such as field-effect transistors, light-emitting diodes and solar cells. ${ }^{1-7}$ Efficient charge transport through the polymer material is an essential requirement for the operation of these devices. One should therefore understand the factors that influence the charge carrier mobility in conjugated polymers. It is currently understood that the mobility depends both on the electric field ${ }^{8-16}$ and the carrier density. ${ }^{9,13,14,16-19}$ The dependence on the electric field is in particular interesting as the region of negative differential mobility at low electric fields is often observed. ${ }^{12,20,21}$ In this paper, we therefore focus on the effect of electric field on the transport.

The mobility of the polymer material is often estimated from the measurements of diode or transistor current-voltage device characteristics. It is however very difficult to extract the effect of the electric field from such measurements. This is due to the fact that nonuniform distribution of carrier density, the electric field and consequently the mobility, is formed in

the devices, while one normally measures just the total current that reaches the electrodes. ${ }^{22}$ Other techniques for the measurement of mobility of organic materials are the time of flight (TOF) and the pulse radiolysis time resolved microwave conductivity (PR-TRMC). It is however sometimes argued that these techniques probe only the fastest carriers. ${ }^{16,22,23}$

On the other hand, in theoretical considerations, certain effects can be included or excluded from the formalism, and their influence therefore separated. Unfortunately, due to complicated structure of these polymer materials, their electronic structure and relevant charge transport parameters are poorly known. Most of the existing theories therefore rely on phenomenological models that assume certain spatial and energetic distribution of states and certain form of the transition rates between them. ${ }^{9,24-27}$ Such models contain several free parameters that should be in principle fitted to the experimental results for the mobility, which are however not always reliable, as discussed above.

In the last few years, significant advances have been made in simulations of transport in disordered organic systems toward the development of parameter free methods. ${ }^{28-36}$ Most of these works, however, analyze the systems consisting of small molecules. We have therefore recently developed a multiscale parameter-free approach for the simulation of charge transport in disordered conjugated polymers. ${ }^{37}$ Here, we extend this approach to include 
the effect of electric field and apply the method to simulate the effect of electric field on hole transport in a fully disordered P3HT polymer. Our methodology therefore serves as a tool that provides an insight into the electric field dependence of the charge transport, complementary to the one obtained from experimental techniques. Our results give new insight into the effect of carrier heating in conjugated polymers - we find, in contrast to previous theoretical considerations based on simplified models, that the concept of effective temperature fails to describe the carrier distribution in the presence of electric field.

\section{THEORY}

The theoretical approach used to simulate the transport in the disordered polymer is based on a multiscale methodology ${ }^{37}$ that consists of several steps that we outline here:

\section{A. Step 1}

Atomic structure of the amorphous P3HT material is obtained from classical molecular dynamics using a simulated annealing procedure. The details of this procedure have been reported in Ref. 38, and will be only briefly outlined here. Five P3HT chains, each 20 rings long, are initially placed in a cubic box much larger than the box corresponding to the density of the system. The temperature is initially set to a value significantly larger than the room temperature and the system is allowed to equilibrate. The size of the box is then gradually decreased down to $29.286 \AA$ which corresponds to experimental density of P3HT. Finally, the system is gradually cooled down to room temperature and subsequently

relaxed to a local minimum. CFF91 force field, ${ }^{39,40}$ modified to properly take into account the interring torsion potentials, ${ }^{38}$ was used, and the simulation was performed using the LAMMPS code. ${ }^{41,42}$ The atomic structure obtained in one such simulation is presented in the left part of Fig. 1.

\section{B. Step 2}

The electronic structure (wave functions and energies of single particle states) of the system obtained in Step 1 was calculated within the framework of density functional theory 
(DFT) in local density approximation (LDA). This was done using our recently developed charge patching method (CPM). ${ }^{38,43}$ Within the CPM, the electronic charge density of the system is constructed by adding together the precalculated charge density motifs assigned to each of the atoms in the system. Charge density motifs are obtained from direct DFT/LDA calculations on small prototype systems where the atoms have the same bonding environment as in the large system. Once the charge density is obtained, one constructs the single particle Hamiltonian $H$ by solving the Poisson equation for the Hartree potential and using the LDA formula for the exchange correlation potential. Several top states in the valence band are then found using the folded spectrum method. ${ }^{44,45}$ The wavefunctions of top 7 of these states for one such calculation are given in the left part of Fig. 1.

\section{C. $\quad$ Step 3}

All phonon modes and electron-phonon coupling constants of the system obtained in Step 1 are then calculated. The phonon modes are calculated by diagonalizing the dynamical matrix

$$
K_{a, b}^{i, j}=\frac{1}{\sqrt{m_{i} m_{j}}} \frac{\partial^{2} E}{\partial x_{a}^{(i)} \partial x_{b}^{(j)}}
$$

obtained from the same classical force field used in molecular dynamics simulation. In Eq. (1), $E$ is the total force field energy, $x_{a}^{(i)}$ is the displacement of atom $i$ in the direction $a$ from its equilibrium position and $m_{i}$ the mass of atom $i$. The eigenvalue problem of the dynamical matrix reads

$$
\sum_{j b} K_{a, b}^{i, j} R_{b}^{(j)}(\nu)=\omega_{\nu}^{2} R_{a}^{(i)}(\nu)
$$

where $\omega_{\nu}$ is the angular frequency of the mode $\nu$. The normal coordinate of mode $\nu$ is given as

$$
v(\nu)=\sum_{i a} R_{a}^{(i)}(\nu) x_{a}^{(i)} \sqrt{m_{i}}
$$

To construct the electron-phonon coupling constants, one needs to find the perturbation of the single particle Hamiltonian $\partial H / \partial x_{a}^{(i)}$ due to the displacement of atom $i$ in the direction $a$, for all $i$ and $a$. This is also done using the CPM, as in step 2. In this case, there is no need to construct the charge density of the perturbed system from the beginning. Since only one atom is moved, one can just use the charge density of the unperturbed system and add to it the change in the motifs of the displaced atom and its neighbors. This makes 
the CPM highly suitable for the efficient computational construction of such perturbation Hamiltonians. From $\partial H / \partial x_{a}^{(i)}$ one obtains the matrix element $\mathcal{N}_{\alpha \beta, a}^{(i)}=\left\langle\alpha\left|\partial H / \partial x_{a}^{(i)}\right| \beta\right\rangle$ between electronic states (obtained in step 2) $|\alpha\rangle$ and $|\beta\rangle$. The electron-phonon coupling element between states $|\alpha\rangle$ and $|\beta\rangle$ due to phonon mode $\nu$ defined as

$$
\mathcal{M}_{\alpha \beta}(\nu)=\langle\alpha|\partial H / \partial v(\nu)| \beta\rangle
$$

can then be obtained as

$$
\mathcal{M}_{\alpha \beta}(\nu)=\sum_{i a} \mathcal{N}_{\alpha \beta, a}^{(i)} \frac{1}{\sqrt{m_{i}}} R_{a}^{(i)}(\nu) .
$$

One can note that in an $N$ atom system (in our case $N=2510$ ), using the straightforward DFT approach (instead of CPM), one would require $3 N$ DFT calculations on an $N$ atom system to calculate the coupling to all phonon modes, which is impossible in practice.

\section{Step 4}

Steps 1-3 give a detailed description of the electronic structure and electron-phonon coupling constants of the portion of material of $\sim 30 \AA$ size. This size is significantly larger than the wave function localization length and therefore each electronic state exhibits a typical environment as in an infinite material in terms of the presence of the other states and phonon modes available for coupling. However, this length scale is too small to obtain the information about the mobility through the bulk polymer material. Therefore, in this step, we construct a larger system consisting of $k_{1} \times k_{1} \times k_{1}$ (where $\left.k_{1}=7\right)$ boxes used in steps 1-3 (referred to as small boxes in the rest of this paragraph), as illustrated in the middle part of Fig. 1. To construct such a system, we repeat $m_{1}=10$ times the calculation performed in steps 1-3 with different initial atomic positions in MD simulation. In addition, we repeat $m_{2}=100$ times steps 1 and 2 , where in step 2 only the valence band maximum (VBM) state is calculated to get a reliable information about the distribution of the VBM states of different small boxes. The larger system is then constructed by randomly choosing one of the $m_{1}$ calculations of steps 1-3 to construct the wavefunctions in each small box. The orientation of the small box is also chosen randomly from one of the permutations of $x, y$ and $z$ axes. The eigenenergies of the states in each of the small boxes are then shifted by the same amount, in such a way to enforce the VBM states of small boxes to obey the calculated distribution (obtained from the $m_{2}$ calculations mentioned previously). Finally, to include 
the effect of the electric field, the eigenenergies of the states are shifted by a linear potential caused by the electric field as

$$
\varepsilon_{\alpha}=\varepsilon_{\alpha}^{(0)}+e \mathbf{F} \cdot \mathbf{r}_{\alpha}
$$

In Eq. (6), $e$ is the absolute magnitude of the charge of an electron, $\varepsilon_{\alpha}^{(0)}$ is the eigenenergy of state $|\alpha\rangle$ in the absence of electric field, $\varepsilon_{\alpha}$ its eigenenergy in electric field $\mathbf{F}$ and $\mathbf{r}_{\alpha}$ the spatial position of state $|\alpha\rangle$ (defined as the center of mass of its probability density distribution).

The transition rates between the states are then calculated using the Fermi's Golden rule expression as $^{37}$

$$
W_{\alpha \beta}=\pi \sum_{\nu} \frac{\left|\mathcal{M}_{\alpha \beta}(\nu)\right|^{2}}{\omega_{\nu}}\left[\left(N_{\nu}+1\right) \delta\left(\varepsilon_{\alpha}-\varepsilon_{\beta}-\hbar \omega_{\nu}\right)+N_{\nu} \delta\left(\varepsilon_{\alpha}-\varepsilon_{\beta}+\hbar \omega_{\nu}\right)\right],
$$

where $N_{\nu}$ is the phonon occupation number given by the Bose-Einstein distribution at a temperature $T$. The $\mathcal{M}_{\alpha \beta}(\nu)$ for the states from the same small box are directly available from the calculation in step 3, while these constants for the states from neighboring boxes need to be approximated, as described in Ref. 37.

We note that Eq. (7) does not take into account the effect of polarons. Recent density functional theory calculations ${ }^{46,47}$ based either on $\mathrm{LDA}^{46}$ or $\mathrm{B} 3 \mathrm{LYP}^{47}$ functionals have shown that polaron binding energy in long straight polythiophene chains is very small - of the order of few meVs only. As a consequence, it has been concluded in Refs. 17,46 that polaron effects could be ignored in practice. It nevertheless remained unclear whether a conclusion based on calculations on ordered polythiophene chains can be extended to disordered polymers. Our calculations (reported in Sec. 5 in Supporting information of Ref. 37) suggested that this is largely the case. It would still be very interesting to use nonperturbative formula for transition rates, and compare to the present results. That will be, however, more computationally demanding, and is beyond the scope of the present work.

Once the transition rates between the states are known, the time evolution of the occupations $n_{\alpha}$ of electronic states (whose positions in space are symbolically marked by small circles in the schematic representation in the middle part of Fig. 1) is given as

$$
\frac{\mathrm{d} n_{\alpha}}{\mathrm{d} t}=-\sum_{\beta} n_{\alpha} W_{\alpha \beta}+\sum_{\beta} n_{\beta} W_{\beta \alpha},
$$

where the first term on the right-hand side represents the total number of carriers leaving the state $\alpha$ in a unit of time and the second term is the number of carriers coming to the 
state $\alpha$ in a unit of time. In the low-carrier density limit that we consider here the effect of Pauli blocking is negligible, and was therefore not included in Eq. (8). We are interested here in the steady state transport when $\frac{\mathrm{d} n_{\alpha}}{\mathrm{d} t}=0$. The system of equations from which the populations of eigenstates can be found reads then

$$
\begin{gathered}
0=-\sum_{\beta} n_{\alpha} W_{\alpha \beta}+\sum_{\beta} n_{\beta} W_{\beta \alpha}, \text { when } \alpha \in\left\{1, \ldots, N_{S}-1\right\} \\
n_{t}=\sum_{\alpha} n_{\alpha},
\end{gathered}
$$

where $N_{S}$ is the total number of states considered and $n_{t}$ their total occupation, which is a predefined small number determined by the carrier density in the system. Appropriate boundary conditions need to be applied to the large $k_{1} \times k_{1} \times k_{1}$ system to close the current flow. This was done in the following manner. The system was firstly periodically replicated in all three directions to form an infinite system. The electric field induced potential was then added according to Eq. (6). In such a way periodic boundary conditions were applied to $\varepsilon_{\alpha}^{(0)}$ and not $\varepsilon_{\alpha}$. These $\varepsilon_{\alpha}$ were then used to calculate the transition rates according to Eq. (7). Periodic boundary conditions for the occupations of all states $n_{\alpha}$ were then imposed. The number of unknown variables in the system of equations (9) is then equal to the number of states in the $k_{1} \times k_{1} \times k_{1}$ system. Such a system of linear equations was then solved. The current through the system was then calculated by choosing a certain reference plane perpendicular to the field direction and calculating the total amount of charge that passes through it in a unit of time. Finally, the mobility was straightforwardly calculated from the current and the total population of states in the system.

\section{E. Step 5}

It turns out that the mobility calculated in step 4 still varies for different random realizations of the system. The calculation in step 4 was therefore repeated $m_{3}=64$ times and such information is used to construct an even larger system containing $k_{2} \times k_{2} \times k_{2}$ (where we take $k_{2}=10$ ) boxes, shown in the right part of Fig. 1. Each of these boxes is considered as an anisotropic conductor corresponding to one of the $m_{3}$ calculations in step 4, randomly chosen and randomly oriented. Its conductivities in the directions perpendicular to the electric field are taken as the conductivities calculated in step 4 in these directions at 
zero electric field, while the conductivity in the direction of the electric field $F$ is naturally taken as the conductivity in that direction at the field $F$. In such a way, a continuum system with spatially varying conductivity is formed. By solving such a system, we obtain the final mobility of the bulk P3HT polymer.

\section{RESULTS AND DISCUSSION}

The dependence of the mobility of disordered P3HT polymer on the electric field calculated using the method described in Sec. II is presented in Fig. 2. The overall trend that we obtain is that the mobility increases with the increase of electric field (positive field dependence) at all temperatures. At higher temperatures there is a slight departure from this trend, as there is a slight decrease of mobility with field (negative field dependence) at low electric fields, as can be seen by careful inspection of Fig. 2.

The dependence of mobility of P3HT polymers on electric field and in particular the sign of its slope, has been discussed in several experimental works. The results of Ref. 48 suggest positive dependence in the case of regiorandom P3HT and negative dependence in regioregular P3HT. In Ref. 12, it was found from TOF measurements that the dependence is positive for temperatures below $250 \mathrm{~K}$, and negative for higher temperatures, in the case of regioregular P3HT. Similar results were reported in Ref. 20, as well. The positive dependence was observed in regiorandom P3HT at room temperature in Ref. 49. Despite the fact that it was shown ${ }^{50}$ that the negative dependence can in some cases be an artifact of the TOF measurement procedure, there seems to be overall consistency among the different experimental results.

Our simulation results of completely amorphous P3HT polymer should be compared to the experimental results on regiorandom P3HT, where it is impossible to establish the ordered structure due to random orientation of alkyl side chains. Therefore, there is an agreement of our results with Refs. 48 and 49 where positive field dependence was also obtained in the samples of regiorandom P3HT. A closer comparison of our simulation results with these results is given in Fig. 3. While there is overall agreement in terms of the order of magnitude of mobility and the overall trends, there are discrepancies both between experimental results from different groups and between experimental results and our simulation. The differences between experimental results can be to some extent assigned to different 
processing conditions and consequently differences in the structure. The differences between theory and experiment can also be assigned to the difference of the atomic structure obtained from MD with the realistic one. Furthermore, it is often argued that TOF techniques probes only the fastest carriers, rather than the steady-state distribution of carriers. ${ }^{16,22,23}$ Whether this is the case, can often be distinguished from the shape of the photocurrent transient signal ${ }^{20}$ in TOF measurements. If the signal is dispersive, then the steady-state distribution is not formed. Dispersive signal has indeed been reported in Ref. 48. There is a question to what extent can the mobility extracted from the dispersive signal be compared to our simulated mobility which probes the carriers obeying the steady-state distribution.

From our simulation, we can further gain insight into the nature of steady-state distribution of carriers at the electric field $F$. Such a distribution for one realization of the system in step 4 is presented in Fig. 4 (bottom part) along with the density of electronic states (top part). The density of states cannot be described by a single Gaussian. Similar result for the density of states has been obtained in recent works on ordered P3HT at room temperature. ${ }^{51,52}$ At low electric fields, the carriers obey the Boltzmann distribution. As the field increases, carriers get hotter, which can be seen from the change in the overall slope of $\ln n$ vs. energy distribution. Additionally, the distribution of carriers starts to deviate from the Boltzmann-like, i.e. the spread from the straight line on the plot becomes larger. Despite these deviations from the straight line, it is tempting to fit the whole distribution with a Boltzmann distribution with a different temperature and introduce an effective temperature obtained from such a fit. The dependence of the effective temperature obtained this way on electric field is presented in Fig. 5.

We would like further to understand the meaning of the effective temperature introduced this way. It is certainly a measure of the degree of carrier heating but can it be used for something else? Can the joint effects of temperature $T$ and electric field $F$ be replaced with the effect of effective temperature $T_{\text {eff }}=f(T, F)$ when carrier transport is concerned? Two studies based on phenomenological models ${ }^{53,54}$ have suggested that this is the case. We will however show here the opposite.

In Fig. 6 we therefore present the dependence of the mobility on the effective electronic temperature $T_{\text {eff }}$. The circles in Fig. 6 do not fall into a single curve, therefore the concept of the effective temperature cannot be used to describe the transport in disordered P3HT material. We would like however to gain a deeper insight into this issue. 
One should note first that not all the hole states in the polymer are relevant for transport. States well below the VBM are certainly not relevant as they are weakly populated. Furthermore, even the states that are occupied can be irrelevant if there are no states in their neighborhood to which carrier can jump. We define the relevant current path according to the following criterion: the connection between states $\alpha$ and $\beta$ is on the relevant current path if $\left|n_{\alpha} W_{\alpha \beta}-n_{\beta} W_{\beta \alpha}\right|>I_{\text {cutt }}$, where $I_{\text {cutt }}$ is chosen such that the relevant path contributes to at least $75 \%$ of the sum of currents at each cross section. The relevant current path for one of the realizations of the system in step 4 at three different electric fields is shown in Fig. 7. At low electric field, the relevant current path is rather sparse, while at higher fields it is quite dense.

Transport properties of the system are clearly mostly determined by the carriers on the relevant current path. One should therefore look at the distribution of states on the relevant current path (rather than all states) to determine whether the Boltzmann-like distribution with an effective temperature is appropriate. The distribution of these states for one realization of the system in step 4 is presented in Fig. 8. It is clear that it is not reasonable to fit such a distribution with a Boltzmann-like one, which contributes to the failure of the concept of effective temperature for the description of transport properties.

In Fig. 9a, we present the histogram of hopping distances in the relevant current path at low electric field and room temperature. This clearly shows that hopping does not take place to nearest neighbors only but it covers a wide range of distances. In the classical Mott's variable-range hopping model ${ }^{55}$ with constant density of states in the region around the Fermi energy and its generalizations, ${ }^{56}$ one can derive analytically ${ }^{56}$ that the concept of effective temperature is valid. In the dilute carrier density limit for the disordered polymer system that we consider here, the density of states in the relevant energy region is certainly not constant and is usually modeled in the literature as the tail of the Gaussian or exponential distribution. Numerical simulations based on such models ${ }^{53,54}$ have also implied that the effective temperature approach is quite satisfactory. It is thus important to understand why our detailed, parameter-free approach gives a qualitatively different result.

In our work, we use the detailed expression that explicitly considers the wave functions of electronic states and their coupling with all phonon modes [Eq. (7)], instead of the simplified Miller-Abrahams ${ }^{16,53,54,57}$ expression. To see the impact of this difference on the results, we have repeated all calculations with a change that the transition probabilities between states 
were modeled according to the Miller-Abrahams formula. The energies of the states were kept the same as in previous calculations. In the Miller-Abrahams expression for downward hops $W=W_{0} \exp (-d / a)$ (where $d$ is the distance between the sites) we have adopted the parameters $W_{0}=10^{14} \mathrm{~s}^{-1}$ and $a=3 \AA$, which also give the hopping distance histogram that covers a range of distances, as demonstrated in Fig. 9b. We note that $W_{0}$ parameter only scales the absolute value of the mobility and cannot change the conclusions related to the concept of the effective temperature. The dependence of the mobility obtained this way on the effective electronic temperature $T_{\text {eff }}$ is shown in squares in Fig. 6. To a reasonably good approximation all squares fall into one curve, which validates the use of effective temperature in the model with Miller-Abrahams hopping rates. Furthermore, in Fig. 8 we show (in squares) the dependence of the occupations of states in the relevant current path on energy. These deviate much less from a Boltzmann distribution than the circles, shown in the same figure. All these results suggest that the difference between our results and phenomenological models arise from the use of simple Miller-Abrahams expression for the transition rates. We have also obtained a similar conclusion from a limited set of calculations with $a=1.5 \AA$ and we further note that the use of the $a$ parameter outside the $1.5-3 \AA$ range yields unrealistically low (high) mobilities, which suggests that these conclusions are not sensitive to the choice of $a$, as long as it is reasonable.

It is also important to gain insight into the energy distribution of carriers that carry the current and the impact of electric field on it. We present the contributions to current of the carriers of different energies in Fig. 10. Such a histogram is obtained in the following way. The energy equal to the average of the energies $\varepsilon_{\alpha}^{(0)}$ and $\varepsilon_{\beta}^{(0)}$ of two states involved is assigned to each transition. The current through a certain plane is then divided into contributions from different transitions and a histogram is formed based on the energies assigned to each of the transitions. Such a histogram is then averaged over several reference planes and over different realizations of the system in step 4, to obtain the histogram given in Fig. 10. As the electric field increases, such a distribution gets a tail from hole states significantly below the VBM. It may seem surprising that the relevant current paths are much more dense at high electric fields than at low electric fields (see Fig. 7), having in mind that at room temperature the mobility increases only by a factor of 2.8 at a field of $1 \mathrm{MV} / \mathrm{cm}$ compared to low electric fields (see Fig. 2). This can be however understood from the results of Fig. 10. At high electric fields, many more states are contributing to the 
current (thus each contributes to a less amount percentage wise), which makes the relevant paths so dense. These contributions are nevertheless not very large and therefore do not lead to a huge increase in mobility.

\section{CONCLUSION}

In conclusion, we have simulated the electric field dependence of hole transport in fully disordered P3HT polymer without the use of any fitting or phenomenological parameters. The results of our simulation that is designed to probe the steady-state DC mobility, yield a similar dependence on electric field as the TOF measurements on regiorandom P3HT samples. Importantly, our simulation gives insight into the nature of nonequilibrium carrier distribution in disordered conjugated polymers at a nonzero electric field. We show that as far as the transport properties are considered, the concept of an effective temperature that replaces the joint effect of electric field and temperature is not valid. This conclusion is essentially different from previous results based on simplified theoretical models, which demonstrates the necessity of applying a detailed simulation framework such as the one presented in this work.

\section{ACKNOWLEDGMENTS}

This work was supported by the DMS/BES/SC of the U.S. Department of Energy under Contract No. DE-AC02-05CH11231. It used the resources of National Energy Research Scientific Computing Center (NERSC). 
1 H. Sirringhaus, P. J. Brown, R. H. Friend, M. M. Nielsen, K. Bechgaard, B. M. W. LangeveldVoss, A. J. H. Spiering, R. A. J. Janssen, E. W. Meijer, P. Herwig, and D. M. de Leeuw, Nature 401, 685 (1999).

2 R. J. Kline, M. D. McGehee, and M. F. Toney, Nature Mater. 5, 222 (2006).

3 J. H. Burroughes, D. D. C. Bradley, A. R. Brown, R. N. Marks, K. Mackay, R. H. Friend, P. L. Burns, and A. B. Holmes, Nature 347, 539 (1990).

4 B. K. Yap, R. Xia, M. Campoy-Quiles, P. N. Stavrinou, and D. D. C. Bradley, Nature Mater. 7, $376(2008)$.

5 N. S. Sariciftci, D. Braun, C. Zhang, V. I. Srdanov, A. J. Heeger, G. Stucky, and F. Wudl, Appl. Phys. Lett. 62, 585 (1993).

6 F. Laquai and D. Hertel, Appl. Phys. Lett. 90, 142109 (2007).

7 Y. Kim, S. Cook, S. M. Tuladhar, S. A. Choulis, J. Nelson, J. R. Durrant, D. D. C. Bradley, M. Giles, I. McCulloch, C.-S. Ha, and M. Ree, Nature Mater. 5, 197 (2006).

8 S. V. Rakhmanova and E. M. Conwell, Appl. Phys. Lett. 76, 3822 (2000).

9 W. F. Pasveer, J. Cottaar, C. Tanase, R. Coehoorn, P. A. Bobbert, P. W. M. Blom, D. M. de Leeuw, and M. A. J. Michels, Phys. Rev. Lett. 94, 206601 (2005).

10 C. Vijila, A. Pivrikas, H. Chun, C. Zhikuan, R. Osterbacka, and C. S. Jin, Org. Electron. 8, 8 (2007).

11 S. R. Mohan, M. Joshi, and M. P. Singh, Org. Electron. 9, 355 (2008).

12 A. J. Mozer and N. S. Sariciftci, Chem. Phys. Lett. 389, 438 (2004).

13 L. Fumagalli, M. Binda, D. Natali, M. Sampietro, E. Salmoiraghi, and P. D. Gianvincenzo, J. Appl. Phys. 104, 084513 (2008).

14 S. Scheinert and G. Paasch, J. Appl. Phys. 105, 014509 (2009).

15 N. Tessler, Y. Preezant, N. Rappaport, and Y. Roichman, Adv. Mater. 21, 1 (2009).

16 V. Coropceanu, J. Cornil, D. A. da Silva Filho, Y. Olivier, R. Silbey, and J.-L. Bredas, Chem. Rev. 107, 926 (2007).

17 R. Coehoorn, W. F. Pasveer, P. A. Bobbert, and M. A. J. Michels, Phys. Rev. B 72, 155206 (2005).

18 C. Tanase, E. J. Meijer, P. W. M. Blom, and D. M. de Leeuw, Phys. Rev. Lett. 91, 216601 
(2003).

19 C. Tanase, E. J. Meijer, P. W. M. Blom, and D. M. de Leeuw, Org. Electron. 4, 33 (2003).

20 A. J. Mozer, N. S. Sariciftci, A. Pivrikas, R. Österbacka, G. Juška, L. Brassat, and H. Bässler, Phys. Rev. B 71, 035214 (2005).

21 V. Kazukauskas, M. Pranaitis, V. Cyras, L. Sicot, and F. Kajzar, Thin Solid Films 516, 8988 (2008), The Tenth European Conference on Organised Films (ECOF-10).

22 R. J. Kline and M. D. McGehee, Polym. Rev. 46, 27 (2006).

23 D. L. Cheung and A. Troisi, Phys. Chem. Chem. Phys. 10, 5941 (2008).

24 P. M. Borsenberger, L. Pautmeier, and H. Bassler, J. Chem. Phys. 94, 5447 (1991).

25 S. D. Baranovskii, H. Cordes, F. Hensel, and G. Leising, Phys. Rev. B 62, 7934 (2000).

26 V. I. Arkhipov, P. Heremans, E. V. Emelianova, G. J. Adriaenssens, and H. Bassler, Appl. Phys. Lett. 82, 3245 (2003).

27 M. C. J. M. Vissenberg and M. Matters, Phys. Rev. B 57, 12964 (1998).

28 A. Troisi, D. L. Cheung, and D. Andrienko, Phys. Rev. Lett. 102, 116602 (2009).

29 J. Kirkpatrick, V. Marcon, J. Nelson, K. Kremer, and D. Andrienko, Phys. Rev. Lett. 98, $227402(2007)$.

30 S. Athanasopoulos, J. Kirkpatrick, D. Martinez, J. M. Frost, C. M. Foden, A. B. Walker, and J. Nelson, Nano Lett. 7, 1786 (2007).

31 W.-Q. Deng and W. A. Goddard, J. Phys. Chem. B 108, 8614 (2004).

32 F. C. Grozema and L. D. A. Siebeless, Int. Rev. Phys. Chem. 27, 87 (2008).

33 J. J. Kwiatkowski, J. M. Frost, and J. Nelson, Nano Lett. 9, 1085 (2009).

34 K. Hannewald, V. M. Stojanović, J. M. T. Schellekens, P. A. Bobbert, G. Kresse, and J. Hafner, Phys. Rev. B 69, 075211 (2004).

35 N. G. Martinelli, Y. Olivier, S. Athanasopoulos, M.-C. R. Delgado, K. R. Pigg, D. A. da Silva Filho, R. S. Sanchez-Carrera, E. Venuti, R. G. D. Valle, J.-L. Bredas, D. Beljonne, and J. Cornil, ChemPhysChem 10, 2265 (2009).

36 G. Nan, X. Yang, L. Wang, Z. Shuai, and Y. Zhao, Phys. Rev. B 79, 115203 (2009).

37 N. Vukmirović and L.-W. Wang, Nano Lett., Article ASAP, DOI: 10.1021/nl9021539 (2009).

38 N. Vukmirović and L.-W. Wang, J. Phys. Chem. B 113, 409 (2009).

39 M. J. Hwang, T. P. Stockfisch, and A. T. Hagler, J. Am. Chem. Soc. 116, 2515 (1994).

40 J. R. Maple, M.-J. Hwang, T. P. Stockfisch, U. Dinur, M. Waldman, C. S. Ewig, and A. T. 
Hagler, J. Comp. Chem. 15, 162 (1994).

41 http://lammps.sandia.gov.

42 S. J. Plimpton, J. Comp. Phys. 117, 1 (1995).

43 N. Vukmirović and L.-W. Wang, J. Chem. Phys. 128, 121102 (2008).

44 L.-W. Wang and A. Zunger, J. Chem. Phys. 100, 2394 (1994).

45 A. Canning, L. W. Wang, A. Williamson, and A. Zunger, J. Comp. Phys. 160, 29 (2000).

46 K. D. Meisel, H. Vocks, and P. A. Bobbert, Phys. Rev. B 71, 205206 (2005).

47 S. S. Zade and M. Bendikov, Chem. Eur. J. 14, 6734 (2008).

48 S. S. Pandey, W. Takashima, S. Nagamatsu, T. Endo, M. Rikukawa, and K. Kaneto, Jpn. J. Appl. Phys. 39, L94 (2000).

49 K. Genevicius, R. Osterbacka, G. Juska, K. Arlauskas, and H. Stubb, Synthetic Metals 137, 1407 (2003).

50 G. Juška, K. Genevičius, K. Arlauskas, R. Österbacka, and H. Stubb, Phys. Rev. B 65, 233208 (2002).

51 D. L. Cheung, D. P. McMahon, and A. Troisi, J. Am. Chem. Soc. 131, 11179 (2009).

52 D. P. McMahon and A. Troisi, Chem. Phys. Lett. 480, 210 (2009).

53 S. Marianer and B. I. Shklovskii, Phys. Rev. B 46, 13100 (1992).

54 F. Jansson, S. D. Baranovskii, F. Gebhard, and R. Osterbacka, Phys. Rev. B 77, 195211 (2008).

55 N. F. Mott, J. Non-Cryst. Solids 1, 1 (1968).

56 V. I. Arkhipov, E. V. Emelianova, and G. J. Adriaenssens, J. Appl. Phys. 93, 6150 (2003).

57 A. Miller and E. Abrahams, Phys. Rev. 120, 745 (1960). 


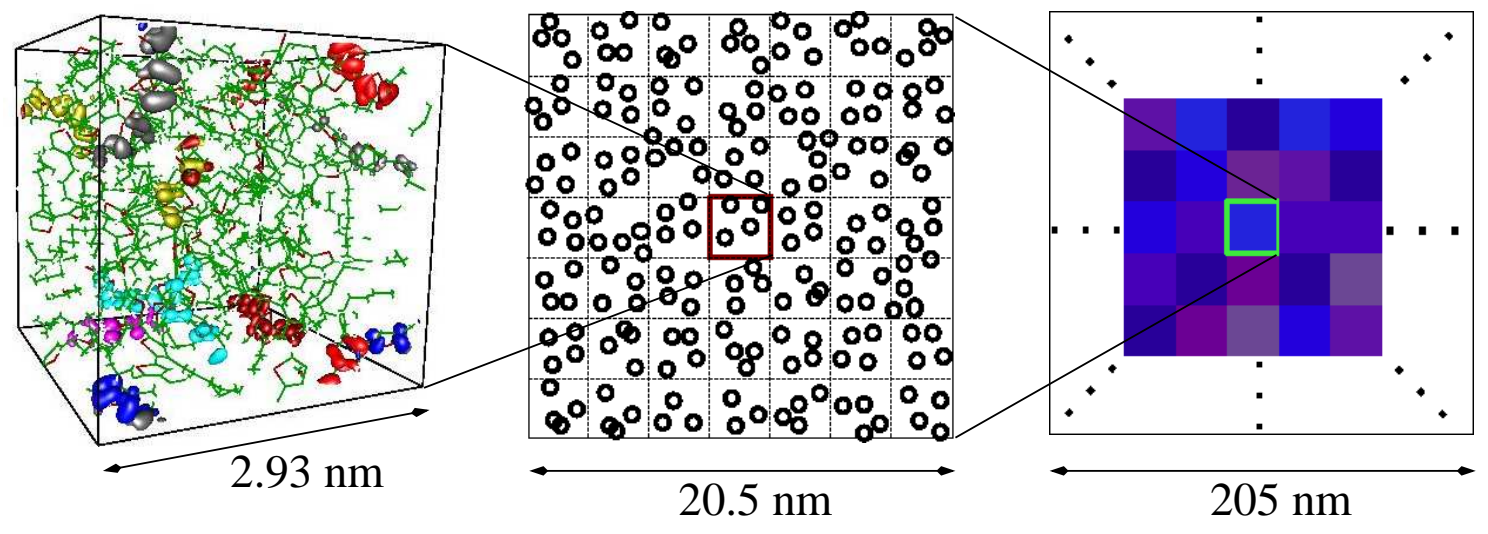

FIG. 1: (Color online) Schematic view of the P3HT polymer system at three different length scales: the atomic structure and the wavefunctions (left), hopping sites (middle) and continuum system (right). 


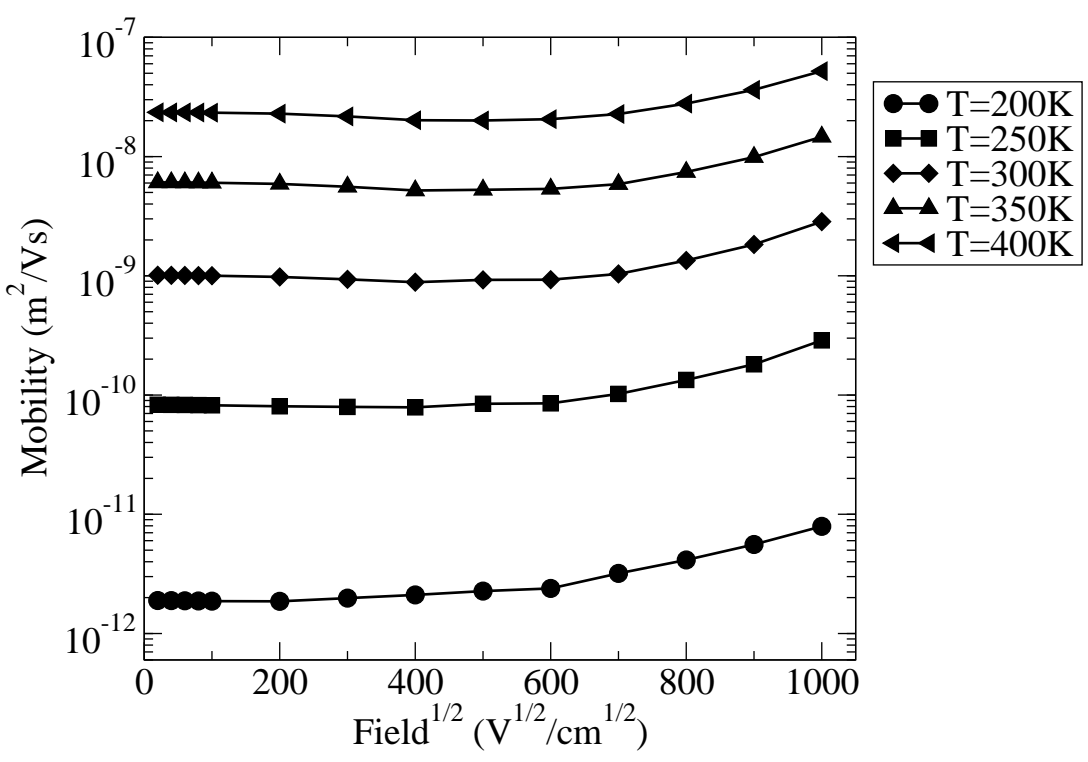

FIG. 2: The dependence of hole mobility in disordered P3HT polymer on electric field at several different temperatures. 


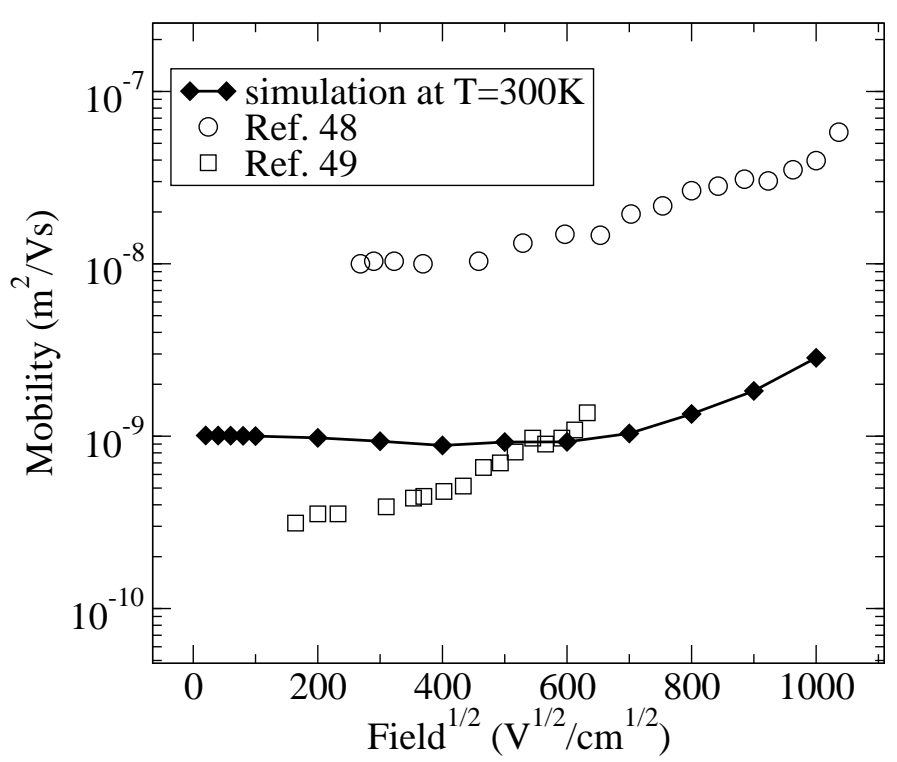

FIG. 3: A comparison of the dependence of hole mobility on electric field at room temperature with experimental measurements of Refs. 48 and 49. 


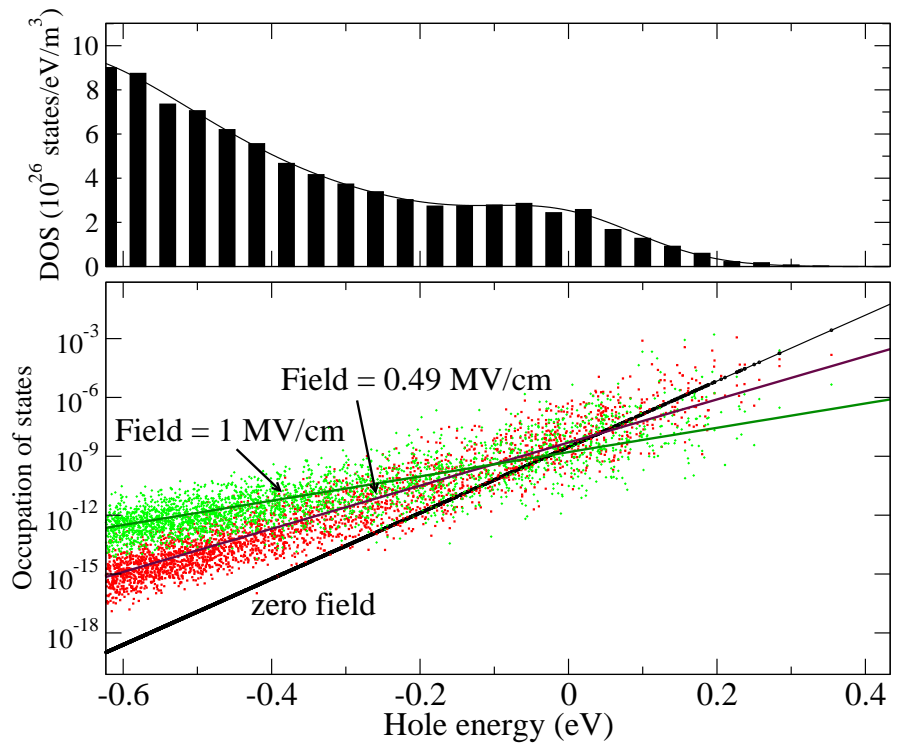

FIG. 4: (Color online) Hole density of states at zero electric field (top). The dependence of the occupation $n_{\alpha}$ of hole states on energy $\varepsilon_{\alpha}^{(0)}$ at several different electric fields and room temperature (bottom). 


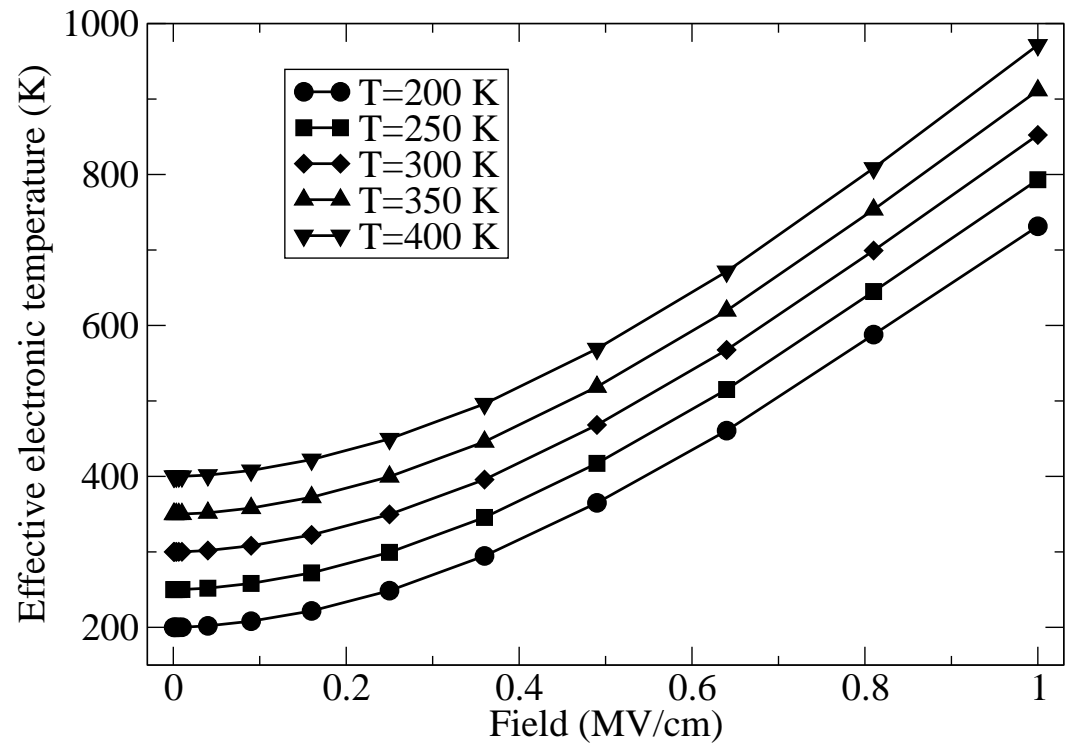

FIG. 5: The dependence of the effective electronic temperature on electric field for several different values of the true lattice temperature. 


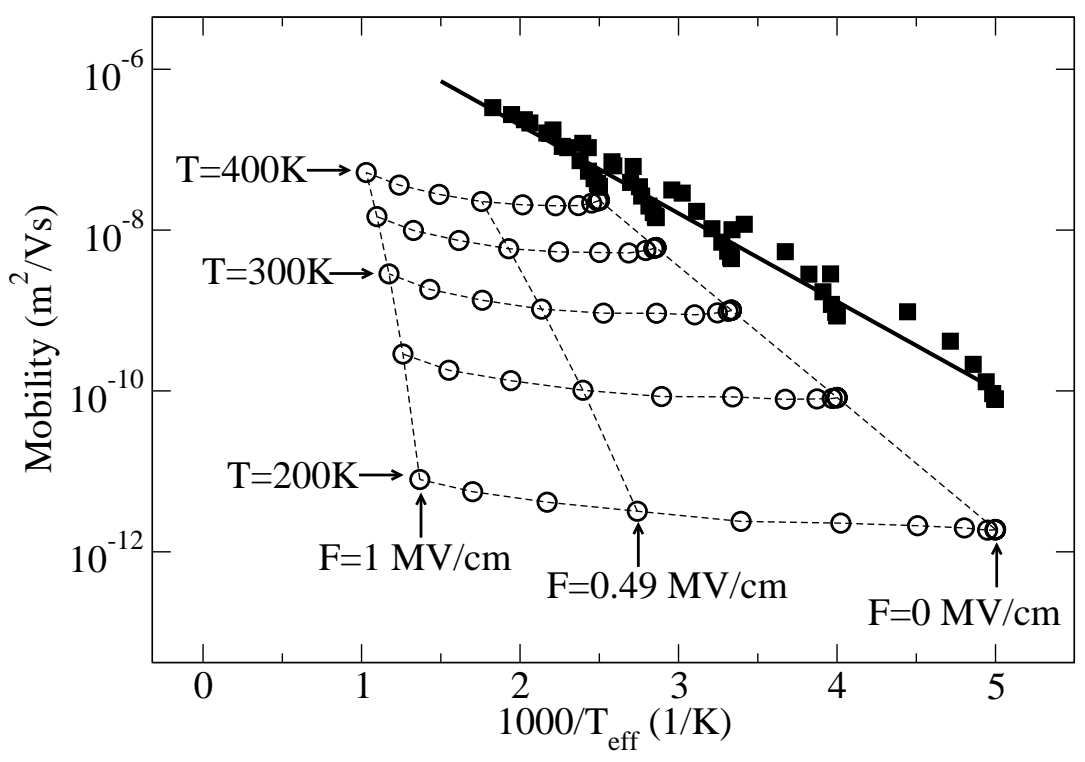

FIG. 6: The dependence of the mobility on the effective electronic temperature $T_{\text {eff. }}$ The calculations done using the method presented in this paper are shown in circles and the calculations done using the same method but with Miller-Abrahams hopping rates are presented in squares. The lines are only guides to the eye. Both sets of calculations have been done for temperatures in the range $200-400 \mathrm{~K}$ and electric field in the range $0-1 \mathrm{MV} / \mathrm{cm}$. 

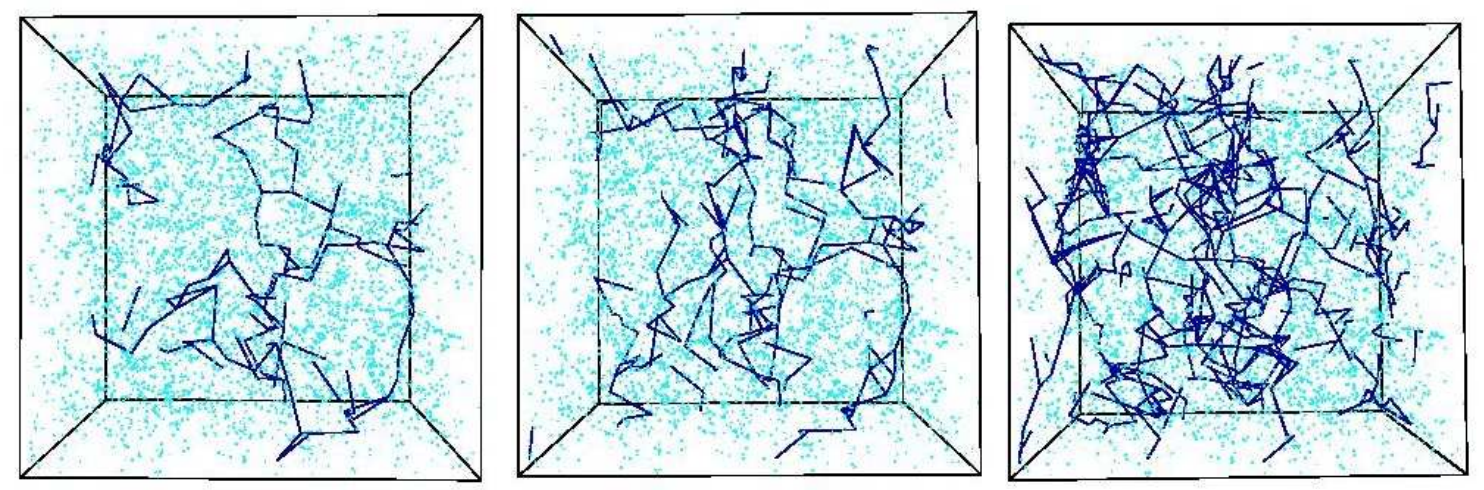

FIG. 7: (Color online) The relevant current paths at room temperature and electric fields of $0,0.3$ and $1 \mathrm{MV} / \mathrm{cm}$, from left to right respectively. The dots represent the states of the system and the lines represent the current paths. 


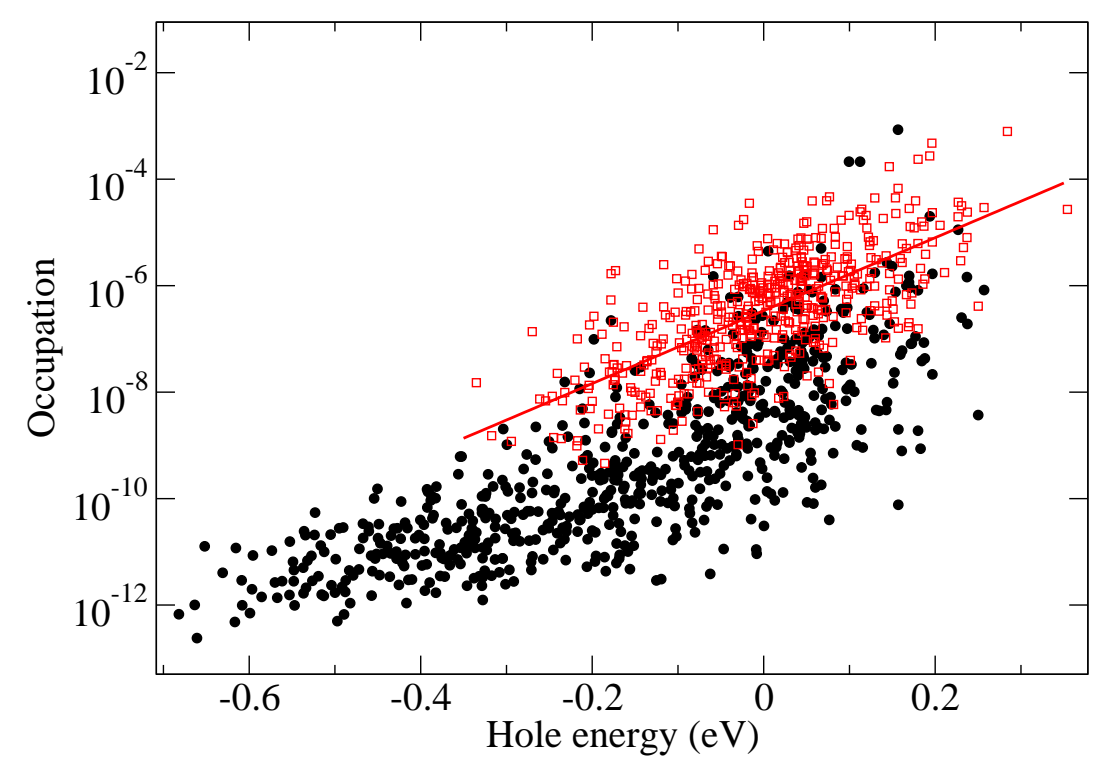

FIG. 8: (Color online) The dependence of the occupation of hole states $n_{\alpha}$ in the relevant current path on energy $\varepsilon_{\alpha}^{(0)}$ at electric field of $1 \mathrm{MV} / \mathrm{cm}$ and room temperature. The results obtained using the methodology presented in this paper are shown in full circles. The results obtained when Miller-Abrahams hopping rates are used instead are shown in squares. The line is only a guide to the eye for these results. 


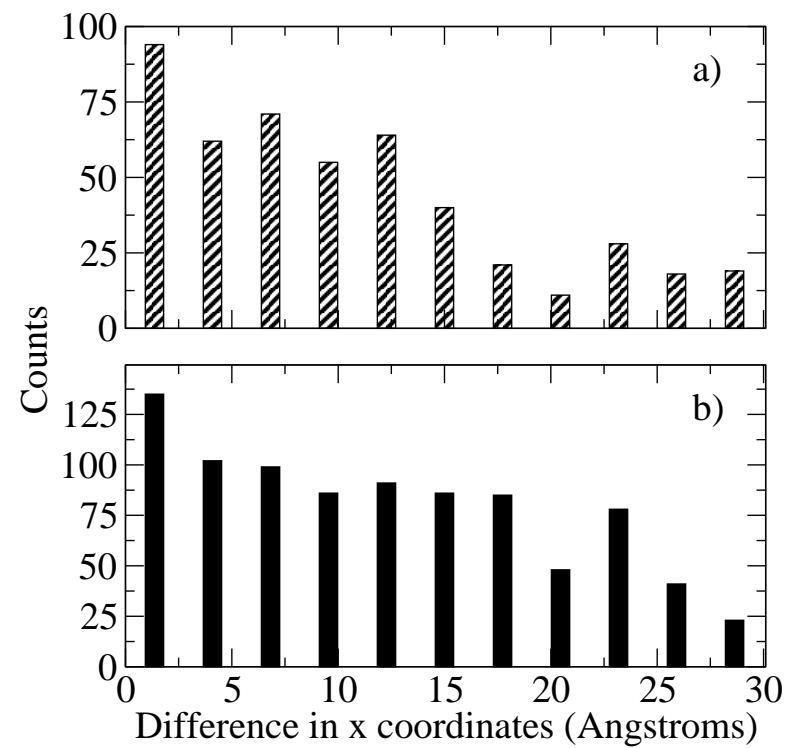

FIG. 9: The histogram of the hopping distances in the $x$ direction for the transitions in the relevant current path when: a) the transition rates are modeled according to Eq. (7); b) the MillerAbrahams model for the transition rates is used. The results are presented for one realization of the $k_{1} \times k_{1} \times k_{1}$ system in step 4 with a small electric field applied in $x$ direction and at room temperature. 

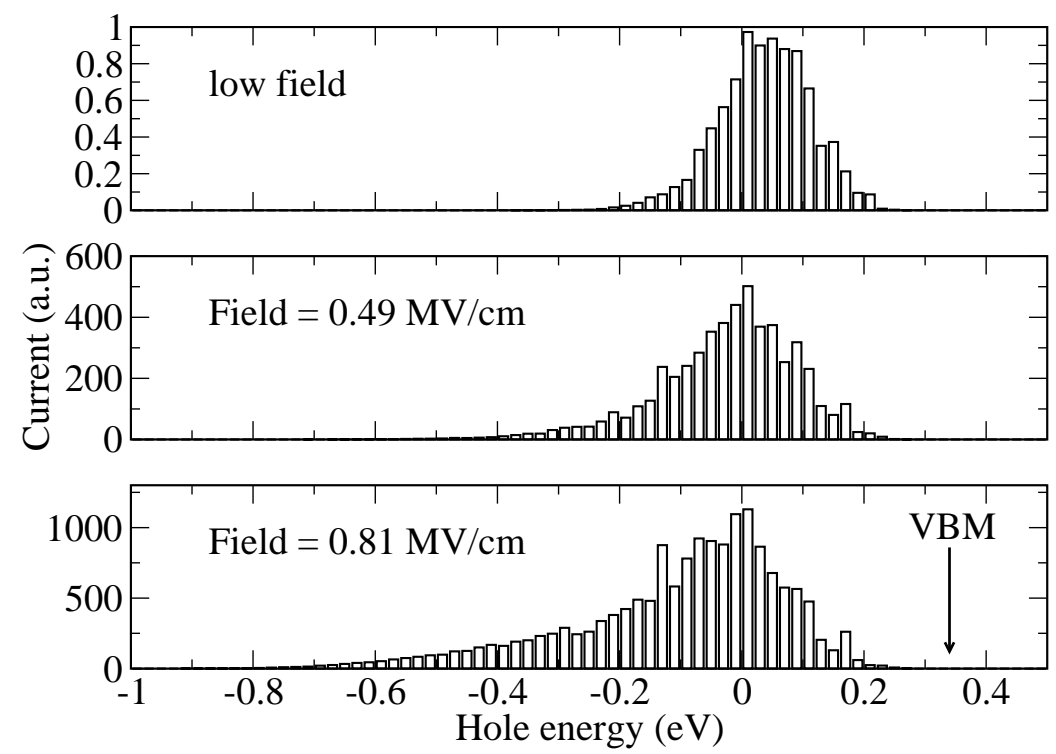

FIG. 10: Energy distribution of the contributions to current at three different electric fields and room temperature. 